\title{
Synchronization and control in time-delayed complex networks and spatio-temporal patterns
}

\author{
S. Banerjee ${ }^{1, \mathrm{a}}$, J. Kurths ${ }^{2,3,4, \mathrm{~b}}$, and E. Schöll ${ }^{5}$ \\ 1 Institute for Mathematical Research, Universiti Putra Malaysia, Selangor, Malaysia \\ 2 Humboldt University, Berlin, Germany \\ 3 Potsdam Institute for Climate Impact Research, 14473 Potsdam, Germany \\ 4 Institute for Complex Systems and Mathematical Biology, University of Aberdeen, \\ Aberdeen, UK \\ ${ }^{5}$ Institut für Theoretische Physik, Technische Universität Berlin, Hardenbergstraße 36, \\ 10623 Berlin, Germany
}

\begin{abstract}
This special topics issue is a collection of contributions on the recent developments of control and synchronization in time delayed systems and space time chaos. The various articles report interesting results on time delayed complex networks; fractional order delayed models; dynamics of spatio-temporal patterns; stochastic models etc. Experimental analysis on synchronization, dynamics and control of chaos are also well investigated using Field Programmable Gate Array (FPGA), circuit realizations and chemical reactions.
\end{abstract}

Complexity and networks, originated in different contexts have been received much attention as interdisciplinary subjects. Of late, they are found to be jointly associated to characterize the so-called complex networked model in different areas that range from natural phenomena on technological aspects to socio-economic systems. It has been reported well that complex networks $(\mathrm{CN})$ widely exist in the real world, including food webs, communication networks, social networks, power grids, cellular and metabolic networks, world wide web, disease transmission networks,neural networks, electrical power grids, scientific citation web, living organisms, etc. $[1,2]$.

Complex networks are involved in a number of different fields that include physics, chemistry, computer science, sociology, economics, finance, biology, epidemiology, etc. However in all of these CN's a very common feature is the presence of a large number of objects (e.g. individuals, computers, cells, molecules, etc.), which interact in a way that quantifies strong irregularities. A network can be essentially represented by a graph, an abstract mathematical structure consists of a set of generically called nodes (vertices) connected by links (edges) representing some binary relationship. In case of World Wide Web, the websites are the nodes and the hyperlinks between

\footnotetext{
a e-mail: santoban@gmail.com

b e-mail: Juergen.Kurths@pik-potsdam.de
} 

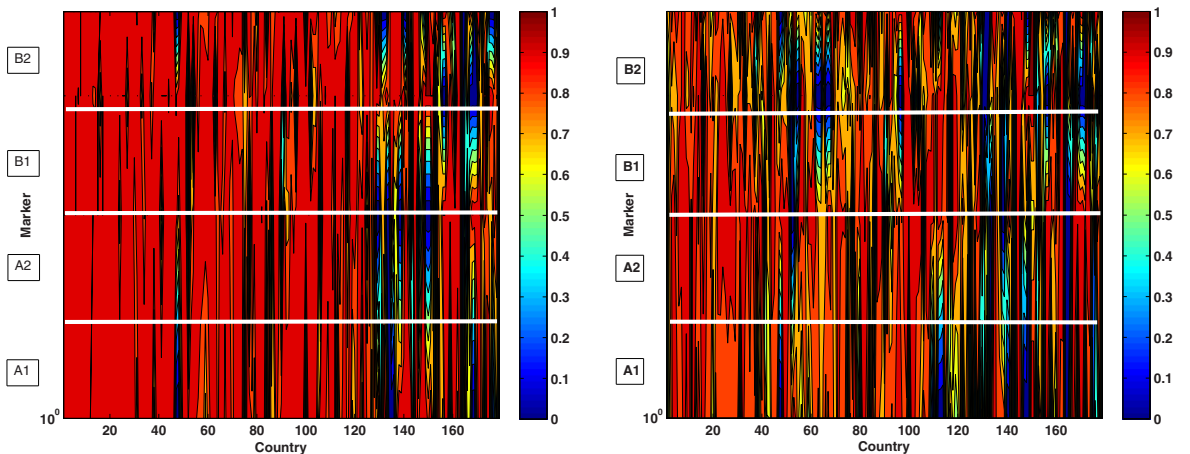

Fig. 1. Contour plot of local clustering coefficient of the A1, A2, B1 and B2 marker GDP data for the year (a) 1990-2015, (b) 2020-2100.

the websites act as link. In Social network the nodes are the people and links exist in form of friendship, professional collaboration, political alliances etc. In Internet, the nodes are the routers and the links represent electrical connections between the nodes. $\mathrm{CN}$ is also used as an alternative tool to understand the dynamical pattern of different real time systems [3]. Recently $\mathrm{CN}$ with time delays are implemented well in synchronization and control problems [4,5].

CN can be constructed from time series and so can also be used in the time series analysis. Once a $\mathrm{CN}$ is constructed from a time series, different statistical measures degree distribution of nodes [7], small world property [6], clustering coefficients and assortativity coefficient [9], degree centrality, eigenvector centrality [9] and such others are used to properly characterize the system generating the time series data. In the past few years, several researches regarding the application of $\mathrm{CN}$ to the study of time series have been [10-12] implemented. Networks formed from chaotic time series are found to possess small world and scale free characteristics, while noisy time series correspond to the characteristics of a random graph [10]. The formation of $\mathrm{CN}$ has also been done from financial time series by many researchers [13]. These types of CN's have the capability to explain different characteristics of the financial data that includes stock returns, global stock markets and securities markets data.

In many large scale networks, sometimes it can be observed that the average distance between the nodes is very small compared to the size of the graphs. The average shortest path (distance between the nodes) length among all possible pair of nodes in the network is known as average distance between vertices. Such large scale networks are said to have small world properties [6]. Small world actually means that it is possible to reach one node from any other in the network through a very small number of intermediate nodes. Thus, small world property exists in a network if the average distance scales logarithmically with the number of nodes. Most of the sociological networks possess this small world property, where it is often called "six degrees of separation".

Figure 1 is an example of clusters in CN. It represent clustering coefficient of different countries under four different marker scenarios. In a more detail, the tendency of forming cliques (triangle) in the complex network constructed on the basis of worldwide GDP data. The more is the value of clustering coefficient, more is the tendency of forming cliques. This ultimately means that the economical policies, political and sociological environment, climate of the countries are more similar that leads to similar GDP. 
The data are the country-level population and Country-level Gross Domestic Product (GDP), which are downscaled projected based on Special Report on Emissions Scenarios (SRES) with A1, A2, B1, B2 marker scenarios. The present SRES GDP projections for individual countries are downscaled out to 2100 by using regional growth rate method, where the regional GDP growth rates were calculated from the marker model (A1, A2, B1, B2) regional data and applied uniformly to each country that fell within the SRES's defined regions. The whole data and information are collected from a Data Centre in NASA's Earth Observing System Data and Information System (EOSDIS) hosted by Center for International Earth Science Information Network (CIESIN) at Columbia University.

For the past several years, increasing investigations on controllability and synchronization have been carried out in different forms of complex networks. Various approaches also have been proposed on how to deal with synchronization in complex networks. Many systematic results have been carried out with respect to the models [18], the methods and the different approaches for handling synchronization of complex networks [19-21]. Among the various analyses, the following are the few recent investigations related to synchronization in complex networks: robustness of synchronization in complex networks; controllability of complex networks; explosive synchronization of complex networks; consensus clustering in complex networks; Recurrence based networks etc.

The concept of deterministic chaos in finite dimensional ODEs has been extended to spatially extended systems described by infinite dimensional PDEs, a system with spatial structures. Spatio-temporal chaos can be observed in Ecological and Biological models, Semiconductor device, Laser and Plasma models [14-17]. Synchronization and control phenomenon can also be investigated well in specially extended systems [22].

This special issue on Synchronization and control in time delayed complex networks and spatio temporal patterns, consists of 19 original regular research articles. Each contribution refers to recent developments of synchronization and control using spatio temporal, time delayed and other systems. Several analytical and experimental results are combined together. The articles can be grouped into four categories, namely 1. Synchronization and control in time delayed and other networks [23-29], 2. Control and synchronization of fractional order systems with time delay [30-32], 3. Dynamics and synchronization: Experiments [33,34], 4. Dynamics of Spatio temporal patterns [35] and 5. Synchronization, control and dynamics of other chaotic models [36-41].

In [23] the author studied scale free networks with Kuramoto oscillators. The existence of a chimera state during cluster explosive transitions to a synchronization regime is also presented.

In [24], the authors studied a model of Hindmarsh-Rose relay neurons with time delay coupling in chain and star topologies. It has been observed that the strength of environmental coupling with the central neuron plays an important role in inducing synchronization and de-synchronization between the outer neurons.

The effect of network-level synchronization with Izhikevich neuron under external periodic signals is reported in [25]. Phase lag synchronization with Izhikevich neuron is also investigated in [26]. The results are useful to study synchronization in complex networks where the nodes are composed by another complex system.

In [27], the authors investigated projective lag synchronization in dynamical networks with identical and non identical nodes. Adaptive control and parameter updating laws have been implemented to design the synchronization. Sufficient conditions for synchronization are also derived analytically.

In [28], the authors studied a stochastic Lorenz system with a modulated time delay. Induced synchronization with white and colored noises are observed between two identical uncoupled systems with unidirectional coupling. This phenomenon 
has been extended to a globally coupled network in the presence of white and color noises.

In [29], the changes in the structure of a neuronal network with the Izhikevich model has been investigated to achieve strong synchronization to simulate episodes of epileptic seizure.

Articles [30-32] are on fractional systems with time delay. In [30] the authors have investigated controllability results for fractional order stochastic dynamical systems with distributed delayed control and Poisson jumps. In [31], the authors presented synchronization network with ring topology using fractional order Lorenz system. A feedback delayed controller has been used to control the system. Numerical results with different parameters are effective to justify the analysis. The analysis in [32] is based on filtering for time delay fractional systems when the constant time-delay is entered in the observation signal. Numerical simulations justify the proposed analysis.

The next section is on experimental observations based on time delayed and other systems [33,34]. In [33], the authors proposed a synchronization scheme and implemented with FPGA analysis. In [34], the authors investigated a novel memristive time delay chaotic system. The dynamics of the system has been investigated using analytical, simulation and experimental observations with circuits.

This research in [35] involves digital mammograms represented in spatial domain and brings out the spatially-localized distributional change in the form of intensity variations over a time period for each patient, which additionally analyzes the temporal data set of current and prior of six different patients with breast cancer. The simulation part is done with real data.

The last section contains dynamical properties, synchronization and control in various stochastic and deterministic models [36-41]. In [36] the dynamical regimes has been investigated, which may be observed in an ensemble of inhibitory coupled Rulkov elements depending on coupling values. Experimental observation have been implemented, where all specified types of neural activity and effects of inhibitory network related to the regimes.

In [37] the complexity of a chaotic systems perturbed by noise and various signals has been investigated using weighted recurrence entropy. Synchronization phenomenon in presence of noise has also been observed by mean conditional recurrence.

In [38], networks of coupled nonlinear oscillators with white Gaussian noise have been investigated analytically. They have shown that noise can prevent phase locking of oscillators, by producing phase diffusion and also the noise can favor synchronization.

In [39] the main aim is to study a new generalized-type of chaos synchronization in continuous-time systems with different dimensions. This type of synchronization occurs between heart and lung, where one can observe that both circulatory and respiratory systems behave in a synchronous way, but their models are essentially different and they have different order.

The coupling and control adaptation of a hybrid electron-nuclear spin system using a laser mediated proton beam in $\mathrm{MeV}$ energy regime has been investigated in [40]. The effect of delay time is also considered in the analysis.

In [41] the authors proposed to implement a NOR gate, a universal Boolean gate, using the BZ reaction by compartmentalizing the reaction into droplets coupled via inhibition. Numerical observations has been done with a chemical model, also experimental results support the analysis.

We would like to thank authors for their contributions and the referees for their efforts in reviewing the manuscripts. We hope this issue will be helpful for the scientists and researchers working on the area of communication and cryptography and various other fields. 
Finally, we would like to thank all members of EPJ ST, for hosting this special issue, specially Dr. Caron, Sabine Lehr, Nicolas and Isabelle for their kind support.

\section{References}

1. J. Cao, Z. Wang, Y. Sun, Phys. A 385, 718 (2007)

2. R. Albert, A.L. Barabási, Rev. Mod. Phys. 74, 47 (2002)

3. R. Albert, A.L. Barabási 85, 5234 (2000)

4. C.P. Li, W.G. Sun, J. Kurths, Phys. A 361, 24 (2006)

5. P. Li, Z. Yi, Phys. A 387, 3729 (2008)

6. M.E.J. Newman, D.J. Watts, Phys. Rev. E 60, 7332 (1999)

7. G. Ghoshal, M.E.J. Newman, Eur. Phys. J. B 58, 175 (2007)

8. M.E.J. Newman, Phys. Rev. Lett. 89, 208701 (2002)

9. M.E.J. Newman, SIAM Rev. 45, 167 (2003)

10. M. Small, J. Zhang, X.K. Xu, Complex Sci. 5, 2078 (2009)

11. N. Marwan, J.F. Donges, Y. Zoua, R.V. Donner, J. Kurthsa, Phys. Lett. A 373, 4246 (2009)

12. J.F. Donges, R.V. Donner, M.H. Trauth, N. Marwan, H.-J. Schellnhuber, J. Kurths, PNAS 108, 20422 (2011)

13. G. Bonanno, G. Caldarelli, F. Lillo, S. Miccieche, N. Vandewalle, R. Mantegna, Phys. J. B 38, 363 (2004)

14. E. Schöll, Nonlinear Spatio-Temporal Dynamics and Chaos in Semiconductors (Cambridge University Press, 2001)

15. S. Banerjee, A.P. Misra, L. Rondoni, Phys. A 391, 107 (2012)

16. S. Banerjee, A.P. Misra, P.K. Shukla, L. Rondoni, Phys. Rev. E 81, 046405 (2010)

17. S. Banerjee, M. Pizzi, L. Rondoni, Opt. Comm. 285, 1341 (2012)

18. F.A. Rodrigues, T.K. DM. Peron, P. Ji, J. Kurths, Phys. Rep. 610, 1 (2016)

19. A. Arenas, A. Diaz-Guilera, J. Kurths, Y. Moreno, C. Zhou, Phys. Rep. 469, 93 (2008)

20. Y. Tang, F. Qian, H. Gao, J. Kurths, Ann. Rev. Control 38, 184 (2014)

21. V. Flunkert, S. Yanchuk, T. Dahms, E. Schöll, Phys. Rev. Lett. 105, 254101

22. L. Lü, C. Li, Y. Wang, W. Wang, Y. Sun, Nonlinear Dyn. 78, 1079 (2014)

23. V. Berec, Eur. Phys. J. Special Topics 225, 7 (2016)

24. U. Singh Thounaojam, P.R. Sharma, M.D. Shrimali, Eur. Phys. J. Special Topics 225, 17 (2016)

25. S. Lobov, A. Simonov, I. Kastalskiy, V. Kazantsev, Eur. Phys. J. Special Topics 225, $29(2016)$

26. V.S.G. Martins, A. Cardoso Rodrigues, H.A. Cerdeira, B. Soares Machado, Eur. Phys. J. Special Topics 225, 41 (2016)

27. Ghada Al-mahbashi, M.S. Md Noorani, S.A. Bakar, M. Mossa Al-sawalha, Eur. Phys. J. Special Topics 225, 51 (2016)

28. S. Majhi, B.K. Bera, S. Banerjee, D. Ghosh, Eur. Phys. J. Special Topics 225, 65 (2016)

29. A.C. Rodrigues, H.A. Cerdeira, B.S. Machado, Eur. Phys. J. Special Topics 225, 75 (2016)

30. T. Sathiyaraj, P. Balasubramaniam, Eur. Phys. J. Special Topics 225, 83 (2016)

31. S. He, K. Sun, H. Wang, Eur. Phys. J. Special Topics 225, 97 (2016)

32. H. Torabi, N. Pariz, A. Karimpour, Eur. Phys. J. Special Topics 225, 107 (2016)

33. S. Sadoudi, C. Tanougast, Eur. Phys. J. Special Topics 225, 119 (2016)

34. V.-T. Pham, S. Vaidyanathan, C.K. Volos, S. Jafari, N.V. Kuznetsov, T.M. Hoang, Eur. Phys. J. Special Topics 225, 127 (2016)

35. P. Shanmugavadivu, V. Sivakumar, R. Sudhir, Eur. Phys. J. Special Topics 225, 137 (2016)

36. T.A. Levanova, A.O. Kazakov, G.V. Osipov, J. Kurths, Eur. Phys. J. Special Topics 225, 147 (2016) 
37. T.S. Dang, S.K. Palit, S. Mukherjee, T.M. Hoang, S. Banerjee, Eur. Phys. J. Special Topics 225, 159 (2016)

38. M. Bonnin, F. Corinto, V. Lanza, Eur. Phys. J. Special Topics 225, 171 (2016)

39. A. Ouannas, M. Mossa Al-sawalha, Eur. Phys. J. Special Topics 225, 187 (2016)

40. V. Berec, Eur. Phys. J. Special Topics 225, 197 (2016)

41. A.L. Wang, J.M. Gold, N. Tompkins, M. Heymann, K.I. Harrington, S. Fraden, Eur. Phys. J. Special Topics 225, 211 (2016) 